\title{
Dynamics of particles around a Schwarzschild-like black hole in the presence of quintessence and magnetic field
}

\author{
Mubasher Jamil ${ }^{a}$, Saqib Hussain, Bushra Majeed \\ School of Natural Sciences (SNS), National University of Sciences and Technology (NUST), H-12, Islamabad, Pakistan
}

Received: 11 August 2014 / Accepted: 13 December 2014 / Published online: 22 January 2015

(C) The Author(s) 2015. This article is published with open access at Springerlink.com

\begin{abstract}
We have investigated the dynamics of a neutral and a charged particle around a static and spherically symmetric black hole in the presence of quintessence matter and external magnetic field. We explore the conditions under which the particle moving around the black hole could escape to infinity after colliding with another particle. The innermost stable circular orbit (ISCO) for the particles are studied in detail. Mainly the dependence of ISCO on dark energy and on the presence of external magnetic field in the vicinity of black hole is discussed. By using the Lyapunov exponent, we compare the stabilities of the orbits of the particles in the presence and absence of dark energy and magnetic field. The expressions for the center of mass energies of the colliding particles near the horizon of the black hole are derived. The effective force on the particle due to dark energy and magnetic field in the vicinity of black hole is also discussed.
\end{abstract}

\section{Introduction}

The accelerating expansion of the universe indicates the presence of elusive dark energy. The presence of dark energy is supported by several astrophysical observations including the study of Ia Supernova [1], cosmic microwave background (CMB) [2] and large scale structure (LSS) [3,4]. The nature of dark energy is not understood until now. It is explained by cosmological models in which dominant factor of dark energy density may possess negative pressure such as cosmological constant $\Lambda$ with a state parameter $w_{q}=-1$. There are other scaler field models that are proposed such as quintessence [5], phantom dark energy [6-9], K-essence [10], holographic dark energy [11-14] to name a few. Dark energy is approximately $70 \%$ of the energy density of the universe. If dark energy is dynamical, then naturally it will become more dominant in the future and will play a crucial

ae-mail: mjamil@sns.nust.edu.pk role at all length scales. In this context, we study the motion of the particles around the black hole surrounded by dark energy and magnetic field.

Observational evidences indicate that magnetic field should be present in the vicinity of black holes [15]. This magnetic field arises due to plasma in the surrounding of black hole. The relativistic motion of particles in the conducting matter in the accretion disk may generate the magnetic field inside the disk. This field does not affect the geometry of the black hole yet, it affects the motion of charged particles and support them to escape [16,17].

Bañados, Silk and West (BSW) proposed that some black holes may act as particle accelerators [18]. In the vicinity of extremal Kerr black hole, they have found that infinite centerof-mass energy (CME) can be achieved during the collision of particles. The BSW effect has been studied for different black hole spacetimes [18-30]. In this paper we obtain the CME expression for the colliding particles near horizon of Schwarzschild-like black hole surrounded by quintessence matter and BSW effect is studied for both neutral and charged particles.

Quintessence is defined as a scalar field coupled to gravity with the potential which decreases as field increases [31]. The solution for a spherically symmetric black hole surrounded by quintessence matter was derived by Kiselev [32]. It has the state parameter in the range, $-1<w_{q}<\frac{-1}{3}$. In this work we will focus on Kiselev solution. We consider a Schwarzschild-like black hole surrounded by quintessence matter in the presence of external axi-symmetric magnetic field. The magnetic field is homogeneous at infinity. This magnetic field and quintessence matter strongly affects the dynamics of the particles and location of their inner stable circular orbits (ISCO) around black hole. During the motion of a particle around the black hole, it is under the influence of dark energy, gravitational and magnetic forces. Before dealing with a difficult problem about dynamics of a charged particle around black hole, we start with the neutral particle 
without considering magnetic field. We construct the dynamical equations from the Lagrangian formalism which are not solvable via analytic methods.

We are extending a previous work [33] by choosing a Schwarzschild-like black hole surrounded by quintessence matter and an external magnetic field. Main objective of our work is to study the effects on the motion of a particle, initially moving in the ISCO, after its collision with another particle. So under what circumstances after the collision the particle would escape from ISCO or its motion would remain bound. Also how would the energy of the particle change after collision. We calculate the force acting on the particle due to dark energy and mention the conditions when this force be attractive or repulsive. In this paper we calculate the velocity of a particle needed to escape to infinity and investigate some characteristics of the particle's motion moving around black hole. We also compare the stability of orbits for photon and massive particle by using Lyapunov exponent [34].

The outline of the paper is as follows: We explain our model in Sect. 2 and derive an expression for escape velocity of the neutral particle. In Sect. 3 we give the expression for magnetic field around the black hole and derive the equations of motion for a charged particle. In Sect. 4 we give the dimensionless form of the equations. In Sect. $5 \mathrm{CME}$ expressions are derived for colliding particles. In Sect. 6 the Lyapunov exponent is calculated. In Sect. 7 the force on the charged particle is calculated. Trajectories for escape energy and escape velocity of the particle are given in Sect. 8. Concluding remarks are given in Sect. 9. We use $(+,-,-,-)$ sign convention and gravitational units, $c=G=1$.

\section{Dynamics of a neutral particle}

We start with the simpler case of calculating the escape velocity of a neutral particle in the absence of magnetic field. The geometry of static spherically symmetric black hole surrounded by the quintessence matter is given by [32]

$$
\begin{aligned}
& \mathrm{d} s^{2}=f(r) \mathrm{d} t^{2}-\frac{1}{f(r)} \mathrm{d} r^{2}-r^{2} \mathrm{~d} \theta^{2}-r^{2} \sin ^{2} \theta \mathrm{d} \phi^{2}, \\
& f(r)=1-\frac{2 M}{r}-\frac{c}{r^{3 w_{q}+1}} .
\end{aligned}
$$

Here $M$ is the mass of black hole, $c$ is the quintessence parameter and $w_{q}$ has range $-1<w_{q}<\frac{-1}{3}$ while we will focus on $w_{q}=\frac{-2}{3}$. The above metric (1) diverges when $r=0$ which is curvature singularity. For $f(r)=0$ we get two values of $r$ :

$r_{+}=\frac{1+\sqrt{1-8 M c}}{2 c}, \quad r_{-}=\frac{1-\sqrt{1-8 M c}}{2 c}$.
The region $r=r_{-}$corresponds to black hole horizon while $r=r_{+}$represents the cosmological horizon. Therefore, $r_{-}$ and $r_{+}$are the two coordinate singularities in the metric (1). If $8 M c=1$ then we get the degenerate solution for the spacetime at $r_{ \pm}=\frac{1}{2 c}$ and if $8 M c>1$ then horizons do not exist. For very small value of $c, r_{+} \approx \frac{1}{c}$. Further more, we can say that the restriction on $c$, is $c \leq \frac{1}{8 M}$.

We discuss the dynamics of a neutral particle in the Schwarzschild-like back ground defined by (1). There are three constants of motion corresponding (1) in which two of them arise as a result of two Killing vectors [35]

$\xi_{(t)}=\xi_{(t)}^{\mu}=\partial_{t}, \quad \xi_{(\phi)}=\xi_{(\phi)}^{\mu}=\partial_{\phi}$.

where $\xi_{t}^{\mu}=(1,0,0,0)$ and $\xi_{\phi}^{\mu}=(0,0,0,1)$ Eq. (3) implies that, black hole metric (1) is invariant under time translation and rotation around symmetry axis $(\theta=0)$. The corresponding conserved quantities (conjugate momenta) are the energy per unit mass $\mathcal{E}$ and azimuthal angular momentum per unit mass $L_{z}$, respectively given by

$\mathcal{E} \equiv f(r) \dot{t}$,

$-L_{z} \equiv \dot{\phi} r^{2} \sin ^{2} \theta$.

Here over dot represents differentiation with respect to proper time $\tau$. The third constant of motion is the total angular momentum

$L^{2}=r^{4} \dot{\theta}^{2}+\frac{L_{z}^{2}}{\sin ^{2} \theta}=r^{2} v_{\perp}^{2}+\frac{L_{z}^{2}}{\sin ^{2} \theta}$.

Here we denote $v_{\perp} \equiv-r \dot{\theta}_{o}$. By using the normalization condition of 4-velocity $u^{\mu} u_{\mu}=1$ and constants of motion (4) and (5), we get the equation of motion of neutral particle

$\dot{r}^{2}=\mathcal{E}^{2}-\left(1+\frac{L_{z}^{2}}{r^{2} \sin ^{2} \theta}\right) f(r)$.

At the turning points of the moving particles from the trajectories $\dot{r}=0$, hence Eq. (7) gives

$\mathcal{E}^{2}=\left(1+\frac{L_{z}}{r^{2} \sin ^{2} \theta}\right) f(r) \equiv U_{\mathrm{eff}}$,

where $U_{\text {eff }}$ is the effective potential.

Consider a particle in the circular orbit $r=r_{o}$, where $r_{o}$ is the local minima of the effective potential. This orbit exists for $r_{o} \in(4 M, \infty)$. Generally for non-degenerate case $\left(r_{+} \neq r_{-}\right)$the energy and azimuthal angular momentum corresponding to local minima $r_{o}$ are

$$
\begin{aligned}
& L_{z o}=\frac{\sqrt{c r_{o}^{2}-2 M}}{\sqrt{c+\frac{6 M-2 r_{o}}{r_{o}^{2}}}}, \\
& \mathcal{E}_{o}=\frac{2\left(2 M+r_{o}\left(c r_{o}-1\right)\right)^{2}}{r_{o}\left(6 M+r_{o}\left(c r_{o}-2\right)\right)} .
\end{aligned}
$$


For the degenerate case which is defined by $c=\frac{1}{8 M}$ or $r_{+}=r_{-}$. The energy and azimuthal angular momentum corresponding to $r_{o}$ are

$L_{z o}=\frac{\sqrt{\frac{r_{o}^{2}}{8 M}-2 M}}{\sqrt{\frac{1}{8 M}+\frac{6 M-2 r_{o}}{r_{o}^{2}}}}$,

$\mathcal{E}_{o}=\frac{2\left(2 M+r_{o}\left(\frac{r_{o}}{8 M}-1\right)\right)^{2}}{r_{o}\left(6 M+r_{o}\left(\frac{r_{o}}{8 M}-2\right)\right)}$.

The ISCO is defined by $r_{o}=4 M$ which is the convolution point of the effective potential [36]. We have not restricted ourself to this local minima at $r_{o}$ because it depends on the applied condition which we will discuss later in Sect. 8 .

Now consider the particle is in a ISCO and collides with another particle, the later one is coming from the rest position at infinity as a freely falling particle. After collision between particles, three cases are possible for the particles: (i) remain bounded around black hole, (ii) captured by black hole and (iii) escape to infinity. The results depend on the collision process. For small changes in energy and angular momentum, orbit of the particle is slightly perturbed but particle remains bounded. For larger change in energy and angular momentum, it can go away from initial path and could be captured by black hole or escape to infinity.

After the collision particle should have new values of energy and azimuthal angular momentum and the total angular momentum. We simplify the problem by applying the following conditions: $(i)$ the azimuthal angular momentum does not change and $(i i)$ initial radial velocity remains same after collision. Under these conditions only energy can change by which we can determine the motion of the particle. After collision particle acquires an escape velocity $\left(v_{\perp}\right)$ in orthogonal direction of the equatorial plane [15].

After collision the total angular momentum and energy of the particle become (at $\theta=\frac{\pi}{2}$ )

$L^{2}=r_{o}^{2} v_{\perp}^{2}+L_{z}^{2}$

$\mathcal{E}=\left[f(r)\left(1+\frac{\left(L_{z}+r v_{\perp}\right)^{2}}{r^{2}}\right)\right]^{\frac{1}{2}}$.

These new values of angular momentum and energy are greater from their values before collision because during collision colliding particle may impart some of its energy to the orbiting particle. We get the expression (15) for velocity $v$ from Eq. (14) after solve it for $v$.

\section{Dynamics of a charged particle}

We investigate how does the motion of a charged particle is effected by both magnetic field in the black hole exterior and gravitational field. The general Killing vector equation is [37]

$\square \xi^{\mu}=0$,

where $\xi^{\mu}$ is a Killing vector. Equation (16) coincides with the Maxwell equation for 4-potential $A^{\mu}$ in the Lorentz gauge $A_{; \mu}^{\mu}=0$. The special choice [38]

$A^{\mu}=\frac{\mathcal{B}}{2} \xi_{(\phi)}^{\mu}$,

corresponds to the test magnetic field, where $\mathcal{B}$ is the magnetic field strength. The 4-potential is invariant under the symmetries which corresponds to the Killing vectors as discussed above, i. e.,

$L_{\xi} A_{\mu}=A_{\mu, \nu} \xi^{\nu}+A_{\nu} \xi_{, \mu}^{\nu}=0$.

A magnetic field vector is defined as [35]

$\mathcal{B}^{\mu}=-\frac{1}{2} \mathrm{e}^{\mu \nu \lambda \sigma} F_{\lambda \sigma} u_{\nu}$,

where

$\mathrm{e}^{\mu \nu \lambda \sigma}=\frac{\epsilon^{\mu \nu \lambda \sigma}}{\sqrt{-g}}, \quad \epsilon_{0123}=1, \quad g=\operatorname{det}\left(g_{\mu \nu}\right)$.

$\epsilon^{\mu \nu \lambda \sigma}$ is the Levi Civita symbol. The Maxwell tensor is defined as

$F_{\mu \nu}=A_{\nu, \mu}-A_{\mu, \nu}=A_{\nu ; \mu}-A_{\mu ; \nu}$.

For a local observer at rest in the space-time (1),

$u_{0}^{\mu}=\frac{1}{\sqrt{f(r)}} \xi_{(t)}^{\mu}, \quad u_{3}^{\mu}=\frac{1}{\sqrt{r^{2} \sin ^{2} \theta}} \xi_{(\phi)}^{\mu}$.

The other two components $u_{1}^{\mu}$ and $u_{2}^{\mu}$ are zero at the turning point $(\dot{r}=0)$. From Eqs.(19)-(22) we have obtained the magnetic field given below

$\mathcal{B}^{\mu}=\mathcal{B} \frac{1}{\sqrt{f(r)}}\left[\cos \theta \delta_{r}^{\mu}-\frac{\sin \theta \delta_{\theta}^{\mu}}{r}\right]$.

Here we considered magnetic field to be directed along the vertical ( $z$-axis) and $\mathcal{B}>0$.

The Lagrangian of the particle of mass $m$ and electric charge $q$ moving in an external magnetic field of a curved space-time is given by [28]

$\mathcal{L}=\frac{1}{2} g_{\mu \nu} u^{\mu} u^{\nu}+\frac{q A_{\mu}}{m} u^{\mu}$

$v_{\perp}^{\mathrm{esc}} \geq \frac{L_{z} r\left(r-2 M-c r^{2}\right)+\sqrt{r^{4}(r(1-c r)-2 M)\left(2 M+r\left(c r+\mathcal{E}^{2}-1\right)\right)}}{r^{2}(2 M+r(c r-1))}$,

particle would escape if $\left|v_{\perp}^{\text {esc }}\right| \geq v_{\perp}$. 
and generalized 4-momentum of the particle $p_{\mu}=m u_{\mu}+$ $q A_{\mu}$. The new constants of motion are defined below

$\dot{t}=\frac{\mathcal{E}}{f(r)}, \quad \dot{\phi}=\frac{L_{z}}{r^{2} \sin ^{2} \theta}-B$,

here

$B \equiv \frac{q \mathcal{B}}{2 m}$.

By using these constants of motion in the Lagrangian we get the dynamical equations for $\theta$ and $r$, respectively

$$
\begin{aligned}
\ddot{\theta}= & B^{2} \sin \theta \cos \theta-\frac{2}{r} \dot{r} \dot{\theta}-\frac{L_{z}^{2} \cos ^{2} \theta}{r^{4} \sin ^{3} \theta}, \\
\ddot{r}= & -\frac{(2 M+r(c r-1))^{2}}{\left(-2 M+r-c r^{2}\right)}\left(\frac{L_{z}^{2}}{r^{4} \sin ^{2} \theta}\right) \\
& +\frac{\left(2 M+c r^{2}\right)}{\left(2 M+c r^{2}-r\right)^{2}}\left(\mathcal{E}+\dot{r}^{2}\right) \\
& +\frac{(2 M+r(c r-1))^{2}}{\left(-2 M+r-c r^{2}\right)}\left(B^{2} \sin ^{2} \theta+\dot{\theta}^{2}\right) .
\end{aligned}
$$

By using normalization condition we get

$$
\begin{aligned}
\mathcal{E}^{2}= & \dot{r}^{2}+r^{2} f(r) \dot{\theta}^{2} \\
& +f(r)\left[1+r^{2} \sin ^{2} \theta\left(\frac{L_{z}}{r^{2} \sin ^{2} \theta}-B\right)^{2}\right] .
\end{aligned}
$$

From Eq. (29) we can write the effective potential as

$U_{\mathrm{eff}}=f(r)\left[1+r^{2} \sin ^{2} \theta\left(\frac{L_{z}}{r^{2} \sin ^{2} \theta}-B\right)^{2}\right]$.

The above equation is a constraint i.e. if it is satisfied initially, then it is always valid, provided that $\theta(\tau)$ and $r(\tau)$ are controlled by Eqs. (27) and (28).

Let us discuss the symmetries of Eqs. (24)-(29), these equation are invariant under the transformation given below

$\phi \rightarrow-\phi, L_{z} \rightarrow-L_{z}, B \rightarrow-B$

Therefore, without losing the generality, we consider the positive charged particle. The trajectory of a negatively charge particle is related to positive charge's trajectory by transformation (31). If we make a choice $\mathcal{B}>0$ then we will have to study both cases when $L_{z}>0, L_{z}<0$. They are physically different: the change of sign of $L_{z}$ means the change of direction of the Lorentz force on the particle.

System of Eqs. (24)-(29) is invariant with respect to reflection $(\theta \rightarrow \pi-\theta)$. This transformation retains the initial position of the particle and changes $v_{\perp} \rightarrow-v_{\perp}$. Therefore, it is sufficient to consider only the positive value of $v_{\perp}$.

\section{Dimensionless form of the dynamical equations}

Before integrating our dynamical equations of $r$ and $\theta$ numerically we make these equations dimensionless by introducing the following dimensionless quantities [11]:

$$
\begin{aligned}
2 m & =r_{d} \quad \sigma=\frac{\tau}{r_{d}}, \rho=\frac{r}{r_{d}}, \ell=\frac{L_{z}}{r_{d}}, \\
b & =B r_{d}, \quad c_{1}=c r_{d} .
\end{aligned}
$$

Equations (27) and (28) acquire the form

$$
\begin{aligned}
\frac{\mathrm{d}^{2} \theta}{\mathrm{d} \sigma^{2}}= & b^{2} \sin \theta \cos \theta-\frac{2}{\rho} \frac{\mathrm{d} \rho}{\mathrm{d} \sigma} \frac{\mathrm{d} \theta}{\mathrm{d} \sigma}+\frac{\ell^{2} \cos ^{2} \theta}{\rho^{4} \sin ^{3} \theta} \\
\frac{\mathrm{d}^{2} \rho}{\mathrm{d} \sigma^{2}}= & \left(1+c_{1} \rho^{2}-\rho\right) \frac{\ell^{2}}{\rho^{4} \sin ^{2} \theta} \\
& +\frac{\left(1+c_{1} \rho^{2}\right)}{\left(1+c_{1} \rho^{2}-\rho\right)^{2}}\left(\mathcal{E}+\left(\frac{\mathrm{d} \rho}{\mathrm{d} \sigma}\right)^{2}\right) \\
& -\left(1+c_{1} \rho^{2}-\rho\right)\left(b^{2} \sin ^{2} \theta+\left(\frac{\mathrm{d} \theta}{\mathrm{d} \sigma}\right)^{2}\right) .
\end{aligned}
$$

For the equatorial plane the Eq. (33) is obviously satisfied and the Eq. (34) becomes

$$
\begin{aligned}
\frac{\mathrm{d}^{2} \rho}{\mathrm{d} \sigma^{2}}= & \left(1+c_{1} \rho^{2}-\rho\right)\left(\frac{\ell^{2}}{\rho^{4}}-b^{2}\right) \\
& +\frac{\left(1+c_{1} \rho^{2}\right)}{\left(1+c_{1} \rho^{2}-\rho\right)^{2}}\left(\mathcal{E}+\left(\frac{\mathrm{d} \rho}{\mathrm{d} \sigma}\right)^{2}\right) .
\end{aligned}
$$

We have solved the Eq. (35) numerically by using the built in command NDSolve in Mathematica 8.0. We have obtained the interpolating function as a solution of Eq. (35) and we plot the derivative of interpolating function (radial velocity of the particle) as a function of $\sigma$ in Fig. 1.

Equations (29) and (30) become

$$
\begin{aligned}
& \mathcal{E}^{2}=\left(\frac{\mathrm{d} \rho}{\mathrm{d} \sigma}\right)^{2}+\rho^{2}\left(1-c_{1} \rho-\frac{1}{\rho}\right)\left(\frac{\mathrm{d} \theta}{\mathrm{d} \sigma}\right)^{2}+U_{\text {eff }} . \\
& U_{\text {eff }}=\left(1-c_{1} \rho-\frac{1}{\rho}\right)\left[1+\rho^{2}\left(\frac{\ell}{\rho^{2} \sin ^{2} \theta}-b\right)^{2}\right]
\end{aligned}
$$

The energy of the particle moving around the black hole in a orbit of radius $\rho_{o}$ at the equatorial plane $\left(\theta=\frac{\pi}{2}\right)$ is given by

$U_{\mathrm{eff}}=\mathcal{E}_{o}^{2}=\left(1-c_{1} \rho-\frac{1}{\rho}\right)\left[1+\frac{\left(\ell-b \rho_{o}^{2}\right)^{2}}{\rho_{o}^{2}}\right]$.

Solving $\frac{\mathrm{d} U_{\text {eff }}}{\mathrm{d} \rho}=0$ and $\frac{\mathrm{d}^{2} U_{\text {eff }}}{\mathrm{d} \rho^{2}}=0$ simultaneously we calculate $b$ and $\ell$ in term of $\rho$ where

$$
\begin{aligned}
\frac{\mathrm{d} U_{\mathrm{eff}}}{\mathrm{d} \rho}= & \rho^{2}-2 b \ell \rho^{2}-b^{2} \rho^{4}-2 b^{2} \rho^{5}\left(c_{1}-1\right) \\
& +\ell^{2}\left(3+2 \rho\left(c_{1}-1\right)\right)
\end{aligned}
$$




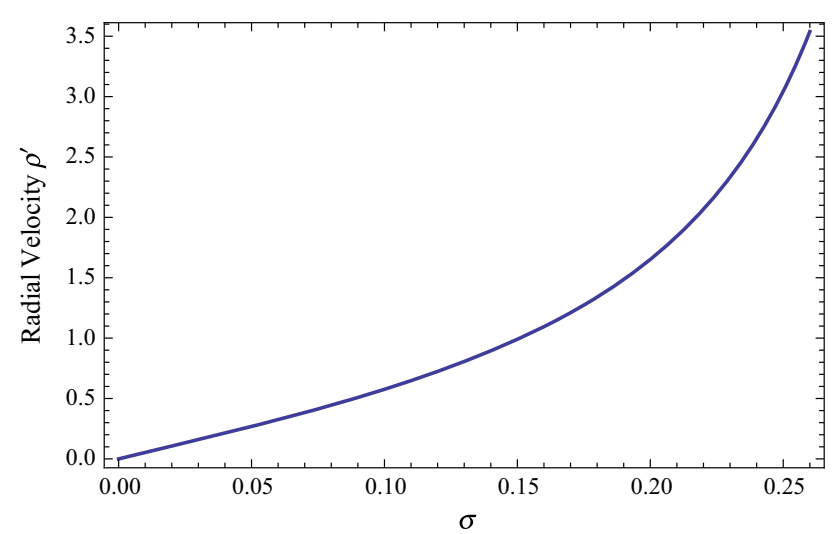

Fig. 1 In this figure we have plotted radial velocity as a function of $\sigma$ for $\ell=5, \mathcal{E}=1, b=0.25$ and $c_{1}=0.125$

and

$$
\begin{aligned}
\frac{\mathrm{d}^{2} U_{\text {eff }}}{\mathrm{d} \rho^{2}}= & 2\left[2 b \ell \rho^{2}-\rho^{2}+b^{2} \rho^{5}\left(1-c_{1}\right)\right. \\
& \left.-3 \ell^{2}\left(2+\rho\left(c_{1}-1\right)\right)\right] .
\end{aligned}
$$

The obtained expressions for $b$ and $\ell$ are

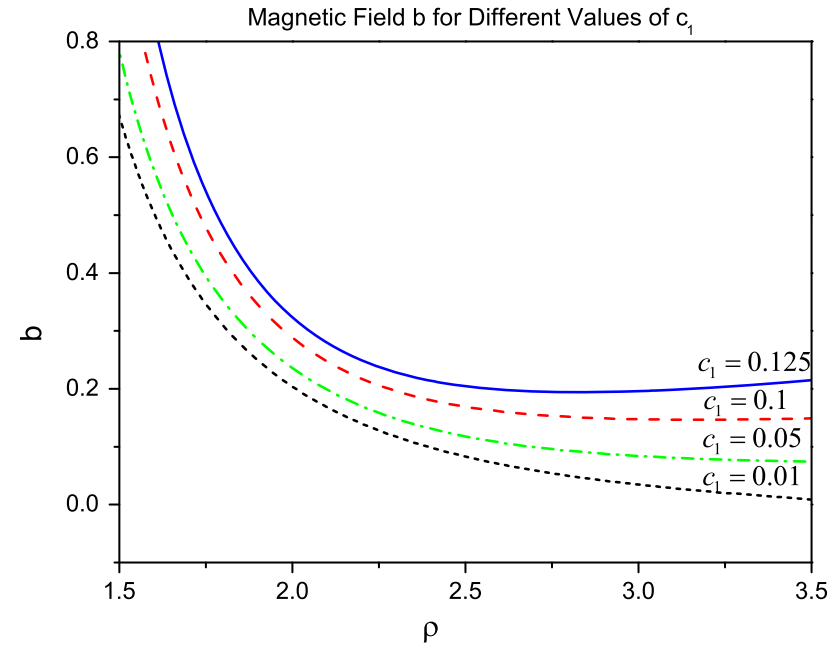

Fig. 2 In this figure we have plotted the magnetic field $b$ as a function of $\rho$ for different value of $c_{1}$

different values of $c_{1}$. Figures 4 and 5 represent the angular momentum for ISCO as a function of magnetic field. Lorentz force is attractive if $\ell>0$ which corresponds to Fig. 4 and it is repulsive if $\ell<0$ corresponds to Fig. 5 .

As we did before in the case of a neutral particle, we assume that the collision does not change the azimuthal

$$
\begin{aligned}
b= & \frac{1}{2 \sqrt{2 \rho^{4}\left(1+\rho\left(c_{1} \rho-1\right)\right)^{2}\left[3+\rho\left(\rho\left(4+c_{1}\left(18+\rho\left(3 c_{1} \rho-18\right)\right)\right)-10\right)\right]}} \\
& \times\left[\sqrt{\rho^{4}\left[1-\rho\left(3+c_{1} \rho\left(\rho\left(3 c_{1} \rho-1\right)-6\right)\right)\right]\left(\rho\left(1+c_{1} \rho\left(6+\rho\left(c_{1} \rho-3\right)\right)\right)-3\right)^{3}}\right. \\
& \left.+\rho^{2}\left(\rho\left(1+c_{1} \rho\left(6+\rho\left(c_{1} \rho-3\right)\right)\right)-3\right)\left(3+\rho\left(\rho\left(4+c_{1}\left(14+3 \rho\left(c_{1} \rho-3\right)\right)\right)-9\right)\right)\right]^{\frac{1}{2}},
\end{aligned}
$$

and

$$
\begin{aligned}
\ell= & \frac{\sqrt{\rho^{4}\left[1-\rho\left(3+c_{1} \rho\left(\rho\left(3 c_{1} \rho-1\right)-6\right)\right)\right]\left(\rho\left(1+c_{1} \rho\left(6+\rho\left(c_{1} \rho-3\right)\right)\right)-3\right)^{3}}}{\left(\rho\left(1+c_{1} \rho\left(6+\rho\left(c_{1} \rho-3\right)\right)\right)-3\right)^{2}} \\
& \times\left[\frac{1}{2 \sqrt{2 \rho^{4}\left(1+\rho\left(c_{1} \rho-1\right)\right)^{2}\left[3+\rho\left(\rho\left(4+c_{1}\left(18+\rho\left(3 c_{1} \rho-18\right)\right)\right)-10\right)\right]}}\right. \\
& \times\left\{\sqrt{\rho^{4}\left[1-\rho\left(3+c_{1} \rho\left(\rho\left(3 c_{1} \rho-1\right)-6\right)\right)\right]\left(\rho\left(1+c_{1} \rho\left(6+\rho\left(c_{1} \rho-3\right)\right)\right)-3\right)^{3}}\right. \\
& \left.\left.+\rho^{2}\left(\rho\left(1+c_{1} \rho\left(6+\rho\left(c_{1} \rho-3\right)\right)\right)-3\right)\left(3+\rho\left(\rho\left(4+c_{1}\left(14+3 \rho\left(c_{1} \rho-3\right)\right)\right)-9\right)\right)\right\}^{\frac{1}{2}}\right] .
\end{aligned}
$$

In Fig. 2 we have plotted magnetic field $b$ against $\rho$ for different values of $c_{1}$. It can be seen that the strength of magnetic field is increasing for large value of $c_{1}$. We can conclude that the presence of dark energy strengthens the magnetic field which is present in the vicinity of black hole. The strength of magnetic field is decreasing away from the black hole. In Fig. 3, we plotted angular momentum as a function of $\rho$ for angular momentum of the particle but it changes the velocity $v_{\perp}>0$. Due to this, the particle energy changes $\mathcal{E}_{o} \rightarrow \mathcal{E}$ and is given by the equation in dimensionless form

$$
\mathcal{E}=\sqrt{\left(\left(1-c_{1} \rho-\frac{1}{\rho}\right)\left[1+\rho^{2}\left(\frac{\ell+\rho v_{\perp}}{\rho^{2}}-b\right)^{2}\right]\right)}
$$




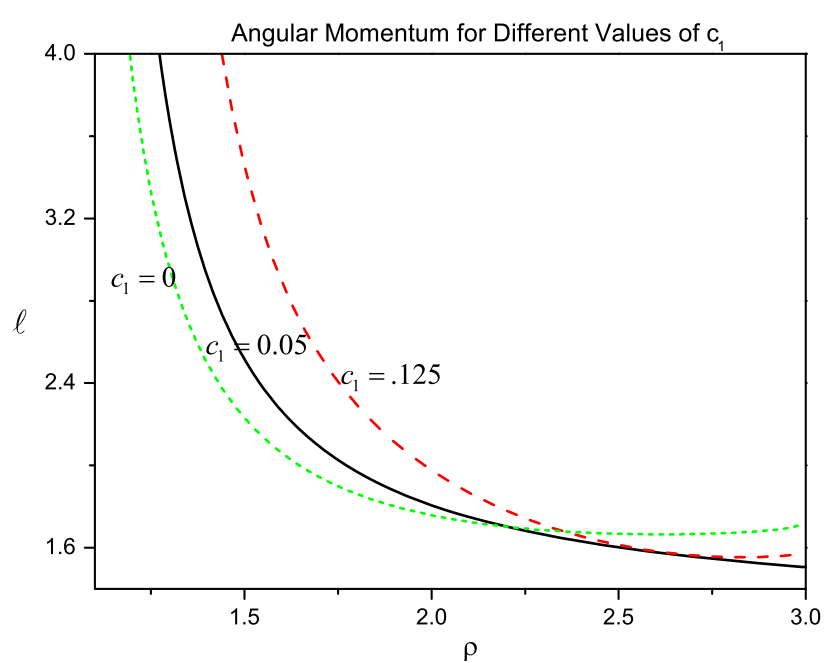

Fig. 3 This figure shows the behavior of angular momentum as a function of $\rho$ for different value of $c_{1}$

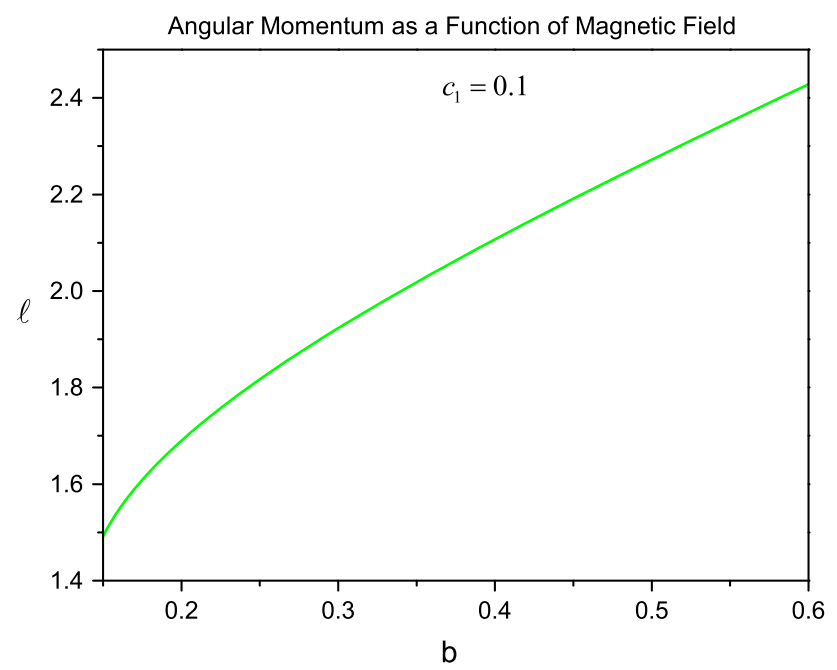

Fig. 4 In this figure we have plotted the angular momentum $\ell_{+}$vs magnetic field $b$

We get escape velocity of the particle from the above equation (43) as given below

$$
\begin{aligned}
v_{\perp}^{\mathrm{esc}} \geq & \frac{1}{\rho^{2}\left(1+\rho\left(c_{1} \rho-1\right)\right.}\left[\rho\left(1+\rho\left(c_{1} \rho-1\right)\right)\left(b \rho^{2}-\ell\right)\right. \\
& \left. \pm \sqrt{\rho^{4}\left(\rho\left(1-c_{1} \rho\right)-1\right)\left(1+\rho\left(\mathcal{E}^{2}+c_{1} \rho-1\right)\right)}\right] .
\end{aligned}
$$

\section{Center of mass energy of the colliding particles}

\subsection{In the absence of magnetic field}

First we consider that the two neutral particles of masses $m_{1}$ and $m_{2}$ coming from infinity collide near the black hole when there is no magnetic field. The collision energy of the

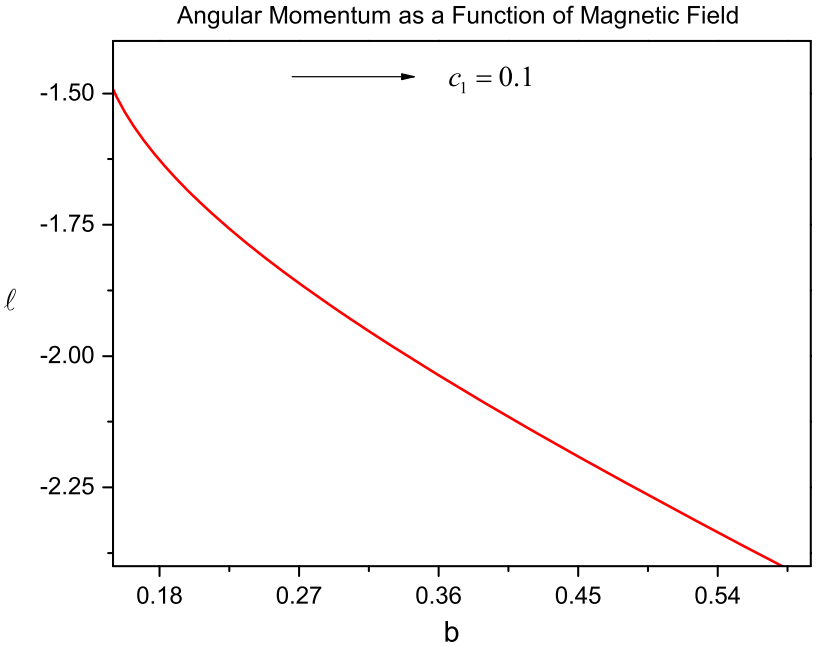

Fig. 5 In this figure we have plotted the angular momentum $\ell_{-}$against magnetic field $b$

particles of masses $m_{1}=m_{2}=m_{o}$ in the center of mass frame is defined as [18]

$E_{c m}=m_{o} \sqrt{2} \sqrt{1-g_{\mu \nu} u_{1}^{\mu} u_{2}^{\nu}}$,

where

$u_{i}^{\mu} \equiv \frac{d x^{\mu}}{d \tau}, \quad i=1,2$

is the 4-velocity of each of the particles. Using Eqs. (4), (5), and (7), in Eq. (45) we get the CME for the neutral particle, falling freely from rest at infinity, given below

$$
\begin{aligned}
E_{c m}= & m_{o} \sqrt{2}\left[2+\left(\frac{L_{1}^{2}+L_{2}^{2}}{2 r^{2}}\right)\left(\frac{\mathcal{E}^{2}+f(r)}{\mathcal{E}^{2}}\right)\right. \\
& \left.+\left(L_{1} L_{2}\right)\left(\frac{f(r)\left(L_{1} L_{2}\right)-2 r^{2} \mathcal{E}^{2}}{2 \mathcal{E}^{2} r^{4}}\right)+\frac{f(r)}{2 \mathcal{E}^{2}}\right]^{1 / 2} .
\end{aligned}
$$

We are interested to find out the CME of the particles near the horizon, so taking $f(r)=0$ we get

$E_{c m}=2 m_{o} \sqrt{1+\frac{1}{4 r_{h}^{2}}\left(L_{1}-L_{2}\right)^{2}}$,

where $r_{h}=\frac{1 \pm \sqrt{1-8 M c}}{2 c}$ represents the horizons of the black hole, obtained earlier. The expression of CME obtained in Eq. (48) could be infinite if the angular momentum of one of the particles gets infinite value, but it would not allow the particle to reach the horizon of the black hole. Thus the CME in Eq. (48) can not be unlimited.

\subsection{In the presence of magnetic field}

For a charged particle moving around the black hole we have obtained the constants of motion defined in Eq. (25), using it 
with normalization condition of the metric we get the equation of motion of the particle

$\dot{r}^{2}=\mathcal{E}^{2}-f(r)\left[1+r^{2}\left(\frac{L}{r^{2}}-B\right)^{2}\right]$.

Using Eqs. (25), (49) with Eq. (45) we get the expression for $\mathrm{CME}$ of the charged particles coming from infinity, colliding near the black hole

$$
\begin{aligned}
E_{c m}= & m_{o} \sqrt{2}\left[2+\left(L_{1}^{2}+L_{2}^{2}\right)\left[\frac{f(r)+\mathcal{E}^{2}}{2 r^{2} \mathcal{E}^{2}}+\frac{f(r) B^{2}}{2 \mathcal{E}^{2}}\right]\right. \\
& -\left(L_{1}+L_{2}\right)\left[B\left(\frac{\mathcal{E}^{2}+f(r)}{\mathcal{E}^{2}}\right)+\frac{f(r) r^{2} B^{3}}{\mathcal{E}^{2}}\right] \\
& +L_{1} L_{2}\left[\frac{f(r)}{2 \mathcal{E}^{2} r^{4}}\left(L_{1} L_{2}+2 B r^{2}\left(L_{1}+L_{2}\right)+4 B^{2} r^{4}\right)\right. \\
& \left.\left.-\frac{1}{r^{2}}\right]+B^{2} r^{2}\left(\frac{\mathcal{E}^{2}+f(r)}{\mathcal{E}^{2}}\right)+\frac{f(r)}{2 \mathcal{E}^{2}}+\frac{f(r) r^{4} B^{4}}{2 \mathcal{E}^{2}}\right]^{1 / 2},
\end{aligned}
$$

near horizon i.e. at $f(r)=0$, Eq. (50) becomes

$E_{c m}=2 m_{o}\left[1+\frac{1}{4 r_{h}^{2}}\left(L_{1}-L_{2}\right)^{2}-\frac{B}{2}\left[\left(L_{1}+L_{2}\right)-B r^{2}\right]\right]^{1 / 2}$,

where $r_{h}$ represent the horizons of the black hole. The CME in Eq. (51) could be infinite, if the angular momentum of one of the particles has infinite value, for which the particle could not reach the horizon of the black hole. Thus the CME defined in Eq. (51) is some finite energy.

\section{Lyapunov exponent for the instability of orbit}

We can check the instability of circular orbit by Lyapunov exponent which is given by [34]

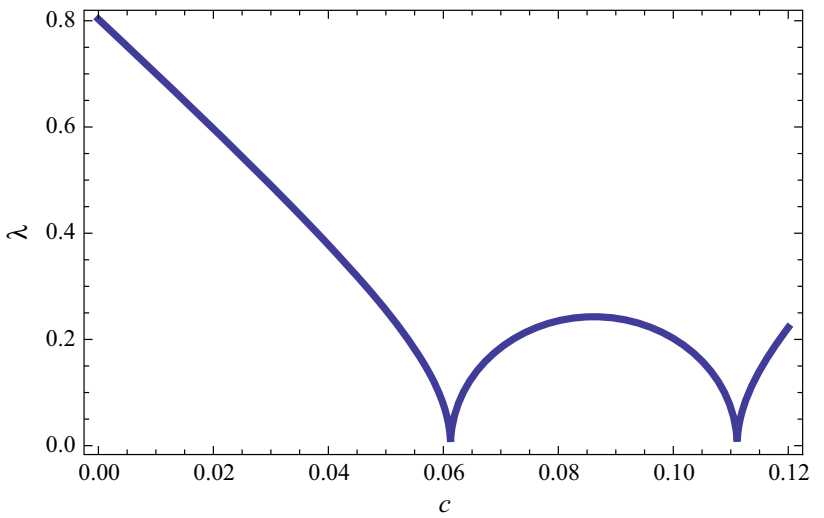

Fig. 6 Figure shows the Lyapunov exponent as a function of $c$ for massive particle. Here, $r=3 M=1, L=3.22$, and $b=0.25$

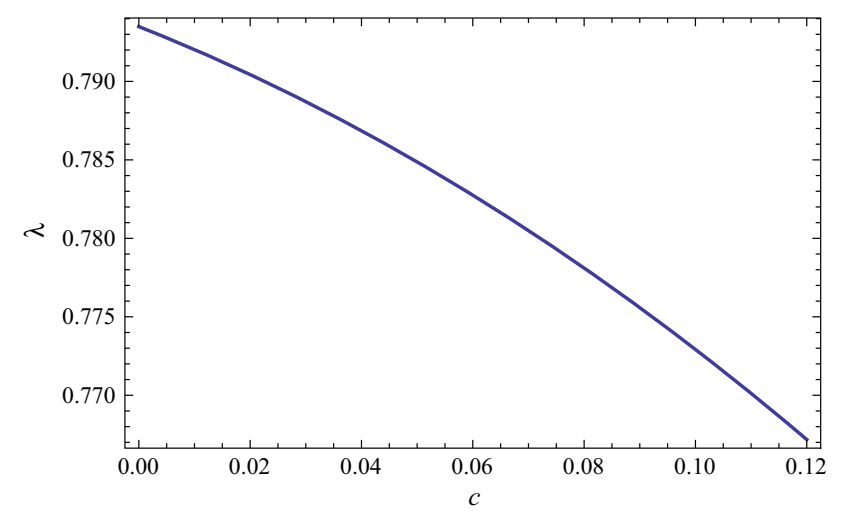

Fig. 7 Figure shows the Lyapunov exponent as a function of $c$ for photon. Here, $r=3, M=1, L=3.22$, and $b=0.25$

immersed in a magnetic field and Schwarzschild-like black hole surrounded by quintessence matter and magnetic field. It can be seen from this figure that with non zero $c$ or $B$, stability is more as compared to Schwarzschild black hole. It can be seen from the Fig. 8 that the Lyapunov exponent $\lambda$ is

$\lambda=\sqrt{\frac{-U_{\text {eff }}^{\prime \prime}\left(r_{o}\right)}{2 \dot{t}\left(r_{o}\right)^{2}}}$
$\lambda=\sqrt{\frac{(2 M+r(-1+c r))\left(-2 M r^{2}+4 B L M r^{2}+B^{2} r^{5}(1-3 c r)-L^{2}(12 M+r(c r-3))\right)}{L^{2} r^{4}}}$

Figures 6 and 7 shows the Lyapunov exponent as a function of $c$. It can be seen form these figures that instability of circular orbits are less for non zero $c$ in comparison with Schwarzschild black hole. In Fig. 8 we are comparing the Lyapunov exponent for three different types of black hole, Schwarzschild black hole, Schwarzschild black hole smaller for the quintessence black hole as compared to $\lambda$ for the stable circular orbit for schwarzschild black hole and it is even more smaller if we consider black hole surrounded by both dark energy and magnetic field. Here for all the figures we denote $b=\frac{B}{r_{d}}$. Figure 9 shows the force acting on the particle as a function of $r$. 


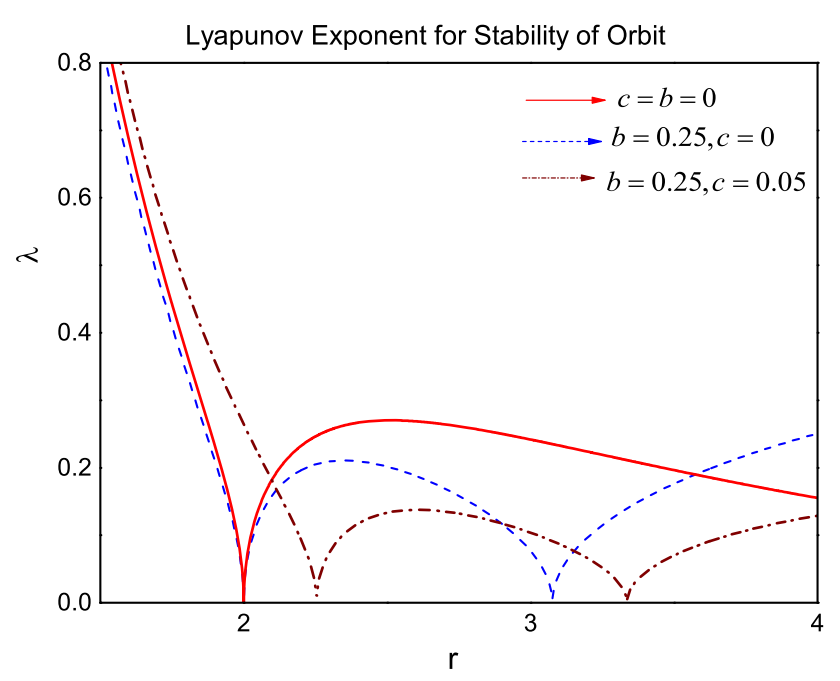

Fig. 8 Lyapunov exponent for different values of $c$ and $b$ as a function of radial coordinate $r$ for $M=1$, and $L=3.22$

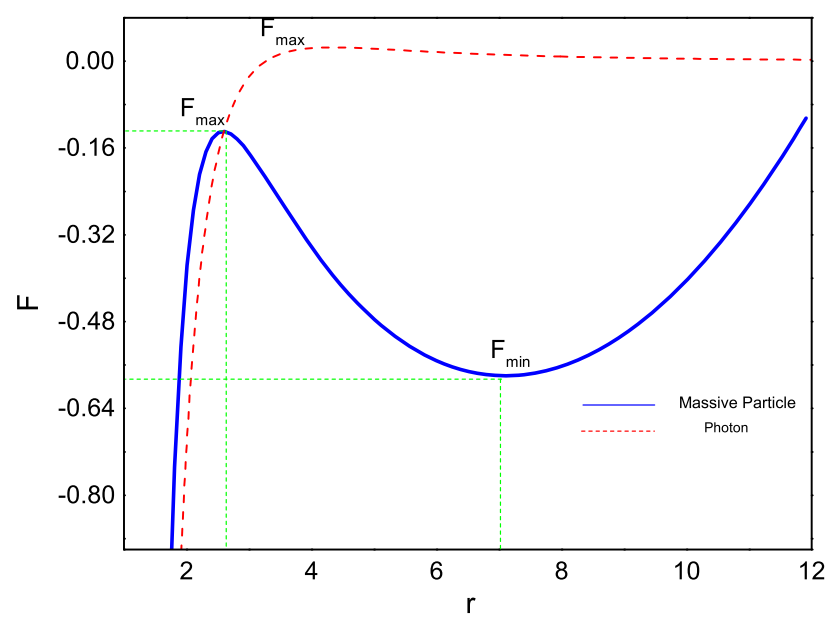

Fig. 9 Figure shows the force on the particle as a function of $r$. Here $c=0.05, M=1, L=3.22$ and $b=0.5$. The orbits corresponds to $F_{\max }$ are unstable and the orbits corresponds to $F_{\min }$ are stable

\section{Effective force on the particle}

We have computed the effective potential, one can obtain the effective force on the particle as

$$
\begin{aligned}
F= & \frac{-1}{2} \frac{\mathrm{d} U_{\text {eff }}}{\mathrm{d} r}, \\
F= & -\frac{M}{2 r^{4}}\left(6 L^{2}-4 B L r^{2}-2 B^{2} r^{4}\right) \\
& +\frac{1}{2 r^{4}}\left(2 L^{2} r-2 r^{2}-2 B r^{5}\right) \\
& -\frac{c}{2 r^{4}}\left(r^{2} L^{2}+2 B L r^{4}-r^{4}-3 B^{2} r^{6}\right)
\end{aligned}
$$

First term in Eq. (55) is attractive if $\left(6 L^{2}>-4 B L r^{2}-2 B^{2}\right.$ $\left.r^{4}\right)$. Second term is repulsive if $\left(2 L^{2} r>-2 r^{2}-2 B r^{5}\right)$. Third term is the force due to quintessence matter, is also attractive if $\left(r^{2} L^{2}+2 B L r^{4}>-r^{4}-3 B^{2} r^{6}\right)$. In case of photon the first term and the dark energy terms are purely attractive (without any condition) and remaining term is repulsive.

For the rotational (angular) variable

$\frac{\mathrm{d} \phi}{\mathrm{d} \tau}=\frac{L_{z}}{r^{2}}-b$.

If the right hand side of Eq. (56) is positive $\left(L_{z}>b\right)$, then the Lorentz force on the particle is repulsive (particle moving in anticlockwise direction). If right hand side is negative ( $L_{z}<$ $b)$, then the Lorentz force is attractive (clockwise rotation).

\section{Trajectories for effective potential and escape velocity}

In Fig. 10 we plotted the effective potential vs $\rho$. The Horizontal line $\alpha$ with $\mathcal{E}<1$ corresponds to bound motion, this is the analogue of elliptical motion in Newtonian theory. The trajectories of the particle is not closed in general. The line segment $\beta$ with $\mathcal{E}>1$ corresponds to a particle coming from infinity and then move back to infinity (hyperbolic motion). The line $\gamma$ does not intersects with the curve of effective potential and passes above its maximum value $U_{1 \max }$. It corresponds to particle which is falling into the black hole (captured by the black hole). In Fig. 10, $U_{1 \text { max }}$ and $U_{2 \max }$ correspond to unstable orbits and $U_{\min }$ refers to a stable circular orbit.

In Fig. 11 we are comparing the effective potentials for different value of $c$. One can notice as the value of $c$ increases the maxima and minima of effective potential shifted downward. Here $U_{\max }$ and $U_{\min }$ corresponds to unstable and stable orbits of the particle around the black hole, respectively. In Fig. 11, curve 3 represents the Schwarzschild effective potential. Therefore, one can say that the dark energy acts to

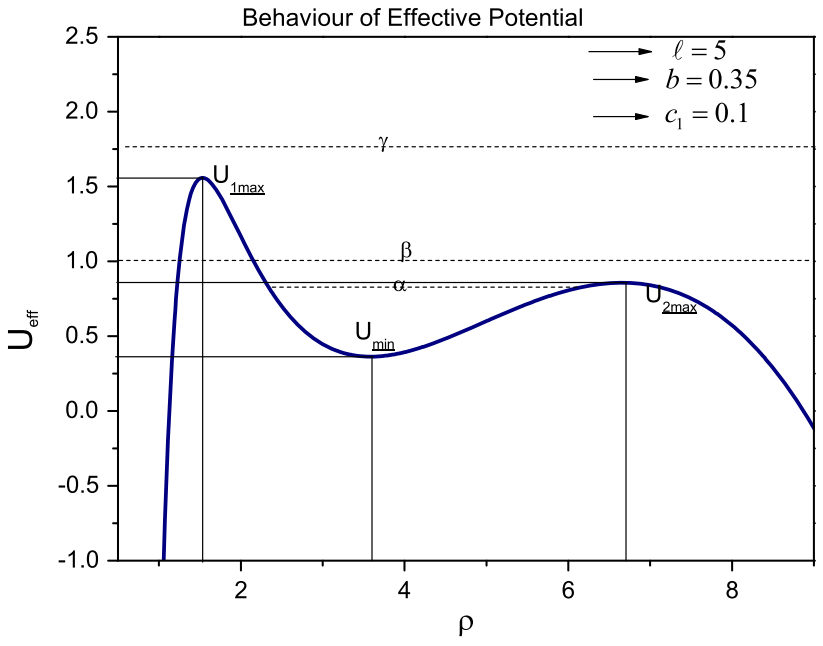

Fig. 10 In this figure we have plotted the effective potential against $\rho$ 


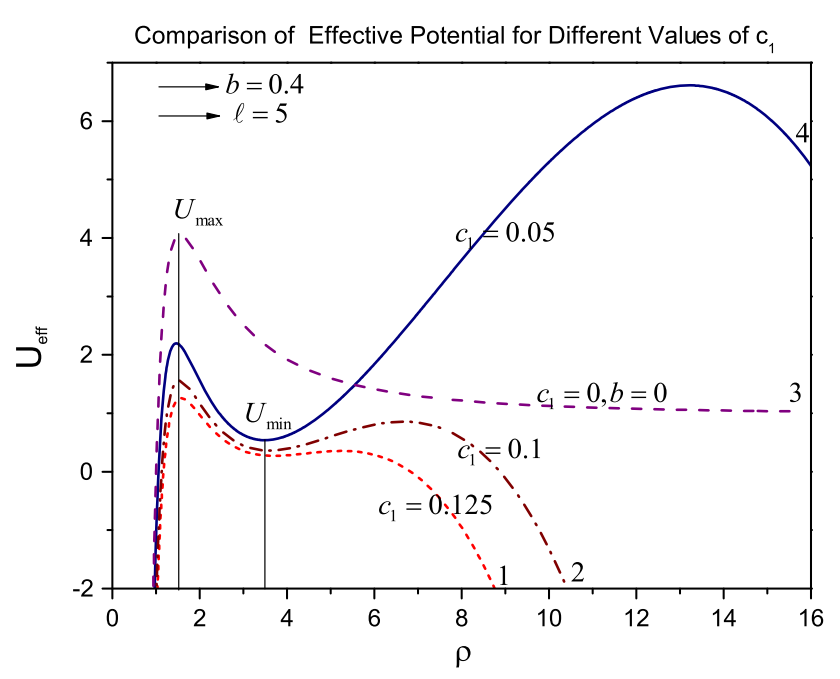

Fig. 11 In this figure we have plotted the effective potential as a function $\rho$, for different value of $c$

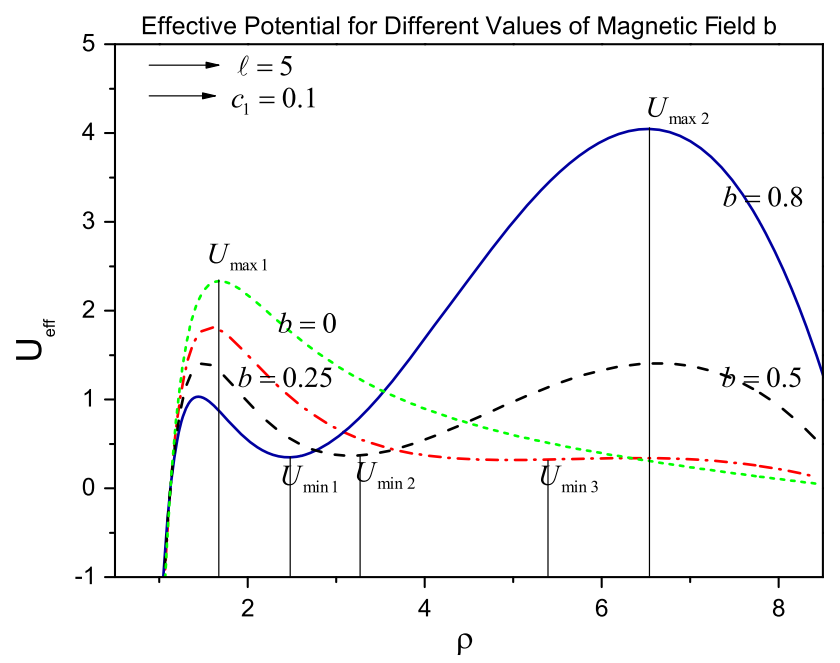

Fig. 12 In this figure we have plotted the effective potential against $\rho$, for different value of magnetic field $b$

decrease the effective potential. We can conclude that force on the particle due to dark energy is attractive. Hence the possibility for a particle to capture by the black hole is greater due to presence of dark energy as compare to the case when $c=0$. Effective potential vs $\rho$ is plotted in Fig. 12 for different values of magnetic field $b$. One can notice from the Fig. 12 that as we increase the strength of magnetic field stability is more as compare to the case for which magnetic field is absent $b=0$. It can also be seen that the local minima of the effective potential which corresponds to ISCO is shifting toward the horizon which is in agreement with $[38,39]$. We are comparing the effective potential for massive particle and photon in Fig. 13. For photon there is no stable orbit as there is no minima for $\ell=0$ represented by plot 3 in Fig. 13 . While for the massive particle there are local minima $U_{\min 1}$ and $U_{\min 2}$ which correspond to stable orbits. It can also be

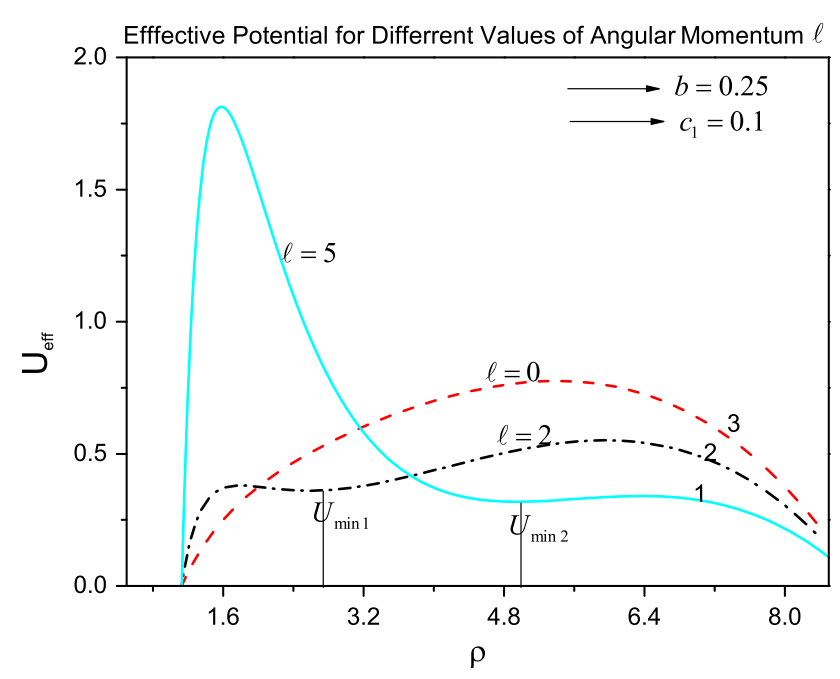

Fig. 13 Here we have plotted the effective potential against $\rho$, for different value of angular momentum $\ell$

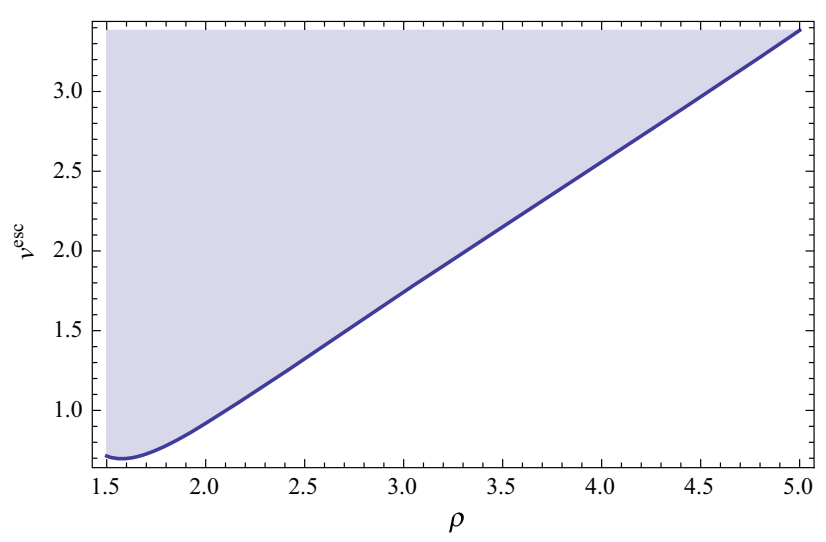

Fig. 14 Here we have plotted the escape velocity as a function of $\rho$ for $\ell=3.22, b=0.50$ and $c_{1}=0.10$

concluded that the particle having larger value of angular momentum $\ell$ can escape easily as compare to the particle with lesser value of angular momentum $\ell$.

Figure 14 explains the behavior of escape velocity of the particle moving around the black hole. In this Fig. 14 the shaded region corresponds to escape velocity of the particle and the solid curved line represents the minimum velocity required to escape from the vicinity of the black hole and the unshaded region is for bound motion around the black hole. In Fig. 15 we have plotted the escape velocity for different values of energy $\mathcal{E}$. Figure 15 shows that the possibility for the particle to escape from the vicinity of black hole having the larger value of energy is greater as compare to the particle with lesser value of energy. Escape velocity for different values of $c_{1}$ is plotted in Fig. 16. This figure shows that the greater the value of $c$ greater will be the escape velocity of the particle provided that the energy of the orbiting particle after collision is less then the $U_{\max }$ otherwise it will captured 


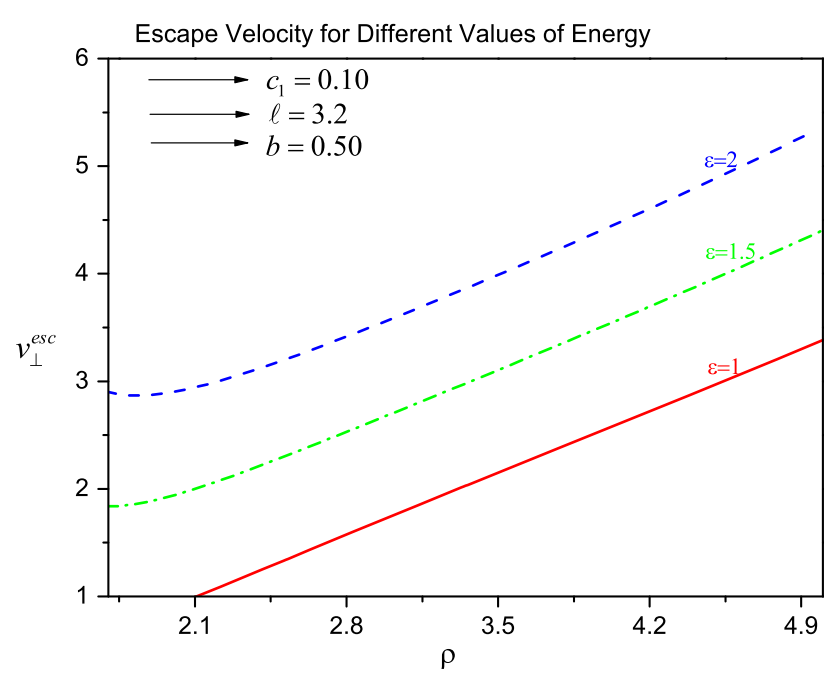

Fig. 15 In this figure we have plotted escape velocity $v_{\text {esc }}$ as a function of $\rho$ for different values of energy $\mathcal{E}$

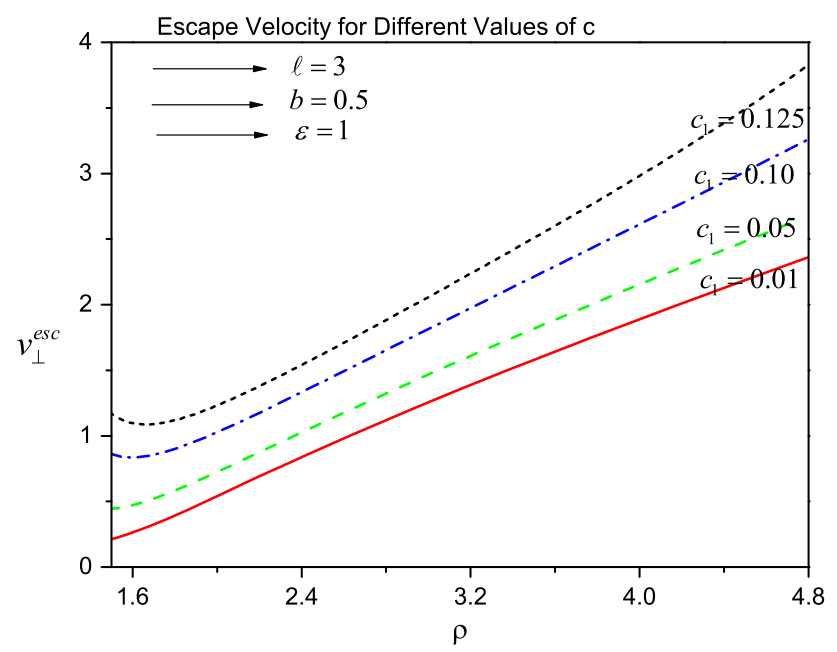

Fig. 16 In this figure we have plotted escape velocity $v_{\text {esc }}$ against $\rho$ for different values of $c_{1}$

by the black hole. One can conclude that the presence of dark energy might play a crucial role in the transfer mechanism of energy to the particle during its motion in the ISCO. In Fig. 17 we are comparing the escape velocity for different values of magnetic field $b$. It can be seen from Fig. 17 that greater the strength of magnetic field the possibility for a particle to escape is more. It can be concluded that the key role in the transfer mechanism of energy to the particle for escape from the vicinity of black hole is played by the magnetic field which is present in the accretion disc. This is in agreement with the result of $[16,17]$.

\section{Summary and conclusion}

- We have studied the dynamics of a neutral and a charged particle in the vicinity of Schwarzschild black hole sur-

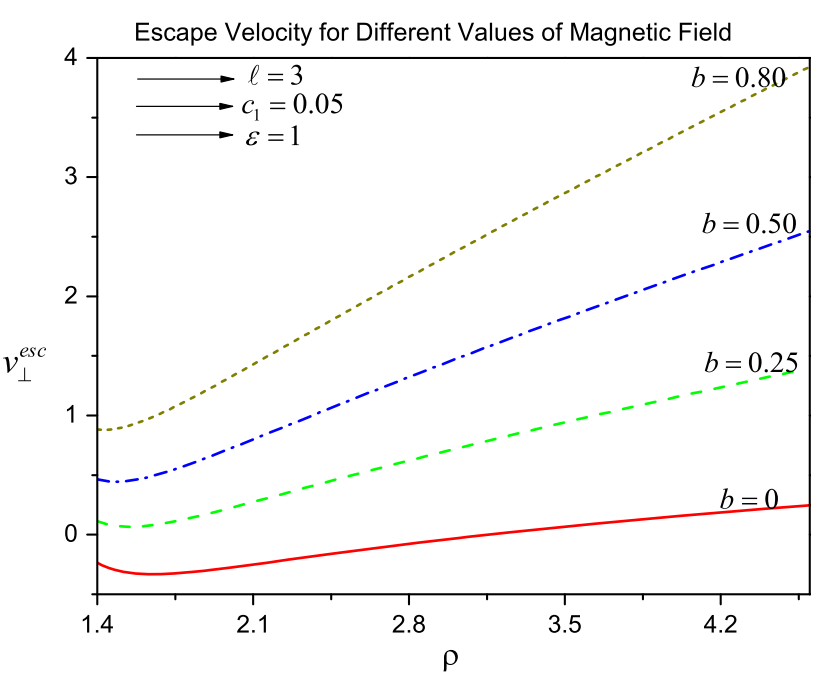

Fig. 17 In this figure we have plotted escape velocity $v_{\text {esc }}$ vs $\rho$ for different values of magnetic field $b$

rounded by quintessence matter. It is known that the quintessence is the candidate for dark energy and the black hole metric which we have studied was derived by Kiselev [32].

- We have studied the motion of a neutral particle in the absence of magnetic field and the dynamics of a charged particle in the presence of magnetic field in the vicinity of black hole in detail.

- We have discussed the energy conditions for the stable circular orbits and for unstable circular orbits around the black hole.

- The ISCO for a massive neutral particle around a Schwarzschild-like black hole occurs at $r=4 \mathrm{M}$.

- We have found that ISCO shifts closer to the event horizon due to presence of dark energy and magnetic field as compare to Schwarzschild black hole. This is the indication that the force due to dark energy is attractive which is agreement with the results of [33].

- Center of mass energy expressions are derived for the colliding particles near horizons. It is found that CME is finite for the particle colliding in the vicinity of Schwarzschild-like black hole surrounded by quintessence.

- We also derived the formula for escape velocity for both particles moving in ISCO.

- The equations of motion have been solved numerically and we plotted the radial velocity of the particle.

- We have calculated the Lyapunov exponent which gives the instability time scale for the geodesics of the particle. Therefore we have concluded that the instability of the circular orbits for Schwarzschild black hole is more in comparison with the black hole which is surrounded by quintessence matter in the presence of magnetic field. 
- We have derived the effective force acting on the particle due to dark energy and mentioned the conditions when the force on the particle due to dark energy is attractive and when it is repulsive.

Acknowledgments The authors would like to thank the reviewer and Azad Akhter Siddiqui for fruitful comments to improve this paper.

Open Access This article is distributed under the terms of the Creative Commons Attribution License which permits any use, distribution, and reproduction in any medium, provided the original author(s) and the source are credited.

Funded by $\mathrm{SCOAP}^{3}$ / License Version CC BY 4.0.

\section{References}

1. S. Perlmutter et al., Astrophys. J. 517, 565 (1999)

2. D.N. Spergel, Astrophys. J. Suppl. 170, 377 (2007)

3. M. Tegmark, Phys. Rev. D 69, 103501 (2004)

4. U. Seljak, Phys. Rev. D 71, 103515 (2005)

5. S.M. Carroll, Phys. Rev. Lett. 81, 3067 (1998)

6. M. Jamil, A. Qadir, Gen. Rel. Grav. 43, 1069 (2011)

7. B. Nayak, M. Jamil, Phys. Lett. B 709, 118 (2012)

8. M. Jamil, D. Momeni, K. Bamba, R. Myrzakulov, Int J. Mod. Phys. D 21, 1250065 (2012)

9. M. Jamil, M. Akbar, Gen. Rel. Grav. 43, 1061 (2011)

10. C. Armendariz-Picon, V. Mukhanov, P.J. Steinhardt, Phys. Rev. Lett. 85, 4438 (2000)

11. S.D.H. Hsu, Phys. Lett. B 594, 13 (2004)

12. M. Li, Phys. Lett. B 603, 1 (2004)

13. A. Pasqua, M. Jamil, R. Myrzakulov, B. Majeed, Phys. Scr. 86, 045004 (2012)

14. B. Majeed, M. Jamil, A.A. Siddiqui, IJTP (2014). doi:10.1007/ s10773-014-2197-3
15. C. Van Borm, M. Spaans, Astron. Astrophys. 553, L9 (2013)

16. J.C. Mckinney, R. Narayan, Mon. Not. R. Astron. Soc. 375, 523 (2007)

17. P.B. Dobbie, Z. Kuncic, G.V.Bicknell, R. Salmeron, Proceedings of IAU Symposium 259 Galaxies (Tenerife, 2008)

18. M. Banados, J. Silk, S. M West, Phys. Rev. Lett. 103, 111102 (2009)

19. O.B. Zaslavskii, JETP Lett. 92, 571 (2010)

20. S.W. Wei, Y.X. Liu, H. Guo, C.E. Fu, Phys. Rev. D 82, 103005 (2010)

21. O.B. Zaslavskii, Phys. Rev. D 81, 044020 (2010)

22. Y. Zhu, S. Wu, Y. Jiang, G. Yang, arXiv:1108.1843

23. M. Patil, A.S. Joshi, K. Nakao, M. Kimura, arXiv: 1108.0288

24. S. Gao, C. Zhong, Phys. Rev. D 84, 044006 (2011)

25. P.J. Mao, R. Li, L.Y. Jia, J.R. Ren, arXiv:1008.2660v3

26. C. Zhong, S. Gao, JETP Lett. 94, 631 (2011)

27. I. Hussain, Mod. Phys. Lett. A 27, 1250017 (2012)

28. I. Hussain, Mod. Phys. Lett. A 27, 1250068 (2012)

29. I. Hussain, J. Phys. Conf. Scr. 354, 012007 (2012)

30. M. Sharif, N. Haider, Astrophys. Space Sci. 346, 111 (2013)

31. E.J. Copeland, M. Sami, S. Tsujikawa, Int. J. Modren. Phys. D 15, 1753 (2006)

32. V.V. Kiselev, Class. Quan. Grav. 20, 1187 (2003)

33. S. fernendo, Gen. Relativ. Gravit. 44, 1857-1879 (2012)

34. V. Cardoso, A.S. Miranda, E. Berti, H. Witech, V.T. Zanchin, Phys. Rev. D 79, 064016 (2009)

35. A.M. Al Zahrani, V.P. Frolov, A.A. Shoom, Phys. Rev. D 87, 084043 (2013)

36. S. Chandrasekher, The Mathematical Theory of Black Holes (Oxford University Press, Oxford, 1983)

37. R.M. Wald, Phys. Rev. D 10, 1680 (1974)

38. A.N. Aliev, N. Ozdemir, Mon. Not. R. Astron. Soc. 336, 241 (1978)

39. G. Prite, Class. Quant. Grav. 21, 3433 (2004) 